\title{
Diffusion-controlled optical elements for optofluidics
}

\section{Citation}

Wolfe, Daniel B., Dmitri V. Vezenov, Brian T. Mayers, George M. Whitesides, Richard S. Conroy, and Mara G. Prentiss. 2005. "Diffusion-Controlled Optical Elements for Optofluidics." Applied Physics Letters 87 (18): 181105. https://doi.org/10.1063/1.2119412.

\section{Permanent link}

http://nrs.harvard.edu/urn-3:HUL.InstRepos:41467476

\section{Terms of Use}

This article was downloaded from Harvard University's DASH repository, and is made available under the terms and conditions applicable to Other Posted Material, as set forth at http:// nrs.harvard.edu/urn-3:HUL.InstRepos:dash.current.terms-of-use\#LAA

\section{Share Your Story}

The Harvard community has made this article openly available.

Please share how this access benefits you. Submit a story.

Accessibility 


\title{
Diffusion-controlled optical elements for optofluidics
}

\author{
Daniel B. Wolfe, Dmitri V. Vezenov, Brian T. Mayers, and George M. Whitesides ${ }^{\mathrm{a}}$ \\ Department of Chemistry and Chemical Biology, Harvard University, 12 Oxford Street, \\ Cambridge, Massachusetts 02138 \\ Richard S. Conroy and Mara G. Prentiss \\ Department of Physics, Harvard University, 17 Oxford Street, Cambridge, Massachusetts 02138
}

(Received 18 May 2005; accepted 30 August 2005; published online 26 October 2005)

\begin{abstract}
Diffusion at the interface between two streams of liquids with different refractive indices, flowing laminarly, creates a controllable concentration gradient and a corresponding refractive index gradient. Using flow rate to change the time over which diffusion occurs in a liquid-liquid $\left(L^{2}\right)$ optical waveguide, we demonstrate an optical splitter and a wavelength filter. The optical splitter comprises two parallel $L^{2}$ waveguides which smoothly merge into a single $L^{2}$ waveguide by diffusion. The wavelength filter comprises an optical splitter in which the two $L^{2}$ waveguides contain an absorbing dye. () 2005 American Institute of Physics. [DOI: 10.1063/1.2119412]
\end{abstract}

This letter describes the controlled use of diffusion to construct an optical splitter and a wavelength filter, where the guiding and cladding elements are both liquids $\left(L^{2}\right.$ waveguides $)^{1}$ flowing at low Reynolds numbers through poly(dimethylsiloxane) (PDMS) microchannels. The optical properties of the liquid optical devices are controlled using the rate of flow and the composition of the fluids. We applied this capability to create both absorbing and nonabsorbing refractive index gradients. These gradients can manipulate light traveling either parallel to the direction of the flow of the liquids, or perpendicular to it. In the case of the optical splitter, light from a single source of white light can be split evenly into the two output channels by a refractive index gradient structure formed by the mutual diffusion of ethylene glycol and water; the same structure, using liquids containing dissolved dyes, split green and red light into separate output channels. We believe these systems establish a new strategy for the fabrication of tunable optical splitters, wavelength and spatial mode filters and other optical devices based on diffusion.

Optical devices such as filters and splitters have been demonstrated in all-solid ${ }^{2-6}$ and liquid-core/solid-cladding waveguides. $^{7}$ Liquid-core/solid-cladding waveguides are convenient and flexible devices for optical filtering because the optical properties (e.g., refractive index, absorption, and fluorescence) of the core can be adjusted easily by changing the liquid (the fluid itself or its solutes). ${ }^{1,7}$ In each of these systems, however, a solid component defines the size and geometry of the optical waveguide (e.g., the angle or location of the split from one waveguide to two or more), and once the device is fabricated, it is, therefore, difficult, if not impossible, to adjust these properties. In addition, the roughness of the interface between the core and cladding materials in these types of waveguides is critical to their optical performance, and achieving appropriate smoothness is expensive and technically difficult.

We demonstrated previously an evanescent coupler based on the mutual diffusion between two $L^{2}$ waveguides. ${ }^{1}$ In this device, light was guided along the path of liquid flow

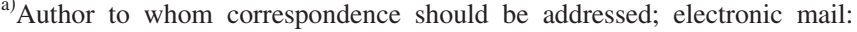
gwhitesides@gmwgroup.harvard.edu (in the direction of increasing extent of diffusive mixing) in two adjacent $L^{2}$ waveguides. The rate of flow determined the length-scale for diffusion and, thus, the refractive index and degree of coupling between the two waveguides.

In the present communication, we describe optical devices based on $L^{2}$ waveguides where the light propagates in a direction opposite to the direction of flow of the fluids, and thus moves in the direction of decreasing extent of diffusive mixing. Two liquid streams, the cores, separated by a narrow (width of $<10 \mu \mathrm{m}$ ) stream of liquid cladding form the light output channels of the splitter. Mutual diffusion of the components of the core and cladding fluids changes the profile of the refractive index across the fluidic channel from that of two separate waveguides at the light output to that of a single waveguide forming the light input channel.

We fabricated the microfluidic channels in poly(dimethylsiloxane) (PDMS) using the standard techniques of soft lithography. ${ }^{8}$ The channel was designed to allow endcoupling of light into the $L^{2}$ waveguide from an optical fiber [Fig. 1(a), and auxiliary information]. The guided light exited the $L^{2}$ waveguides through a transparent, flat window when the guiding fluids were forced to turn by $90^{\circ}$ with a radius of $<0.1 \mathrm{~cm}$ at the T-split [Fig. 1(c)]. ${ }^{9}$ Each end of the channel tapered from a large width $\{500 \mu \mathrm{m}$ at the fluid input [Fig. 1(c)] and $150 \mu \mathrm{m}$ at the fluid output [Fig. 1(b)]\} to a long central region with a small width $(50 \mu \mathrm{m})$. The wide region at the fluid input simplifies the characterization of the light exiting the $L^{2}$ waveguide by expanding the separation between the liquid cores. The wide region at the fluid output improves the coupling efficiency of light from the optical fiber into the $L^{2}$ waveguide, because the core of the optical fiber was $\sim 105 \mu \mathrm{m}$. The narrow region in the center of the channel network [Fig. 1(b) right and 1(c) left] decreases the transverse length over which the core and cladding fluids mix (i.e., the width of the central cladding stream) to $<10 \mu \mathrm{m}$. The light that exits the $L^{2}$ waveguides was imaged through a $10 \times$ microscope objective using a CCD camera. ${ }^{10}$ The light from each output was also coupled from the liquid guide into optical fibers; the coupling efficiency from the input fiber to both of the output optical fibers was $\sim 20 \%$. 


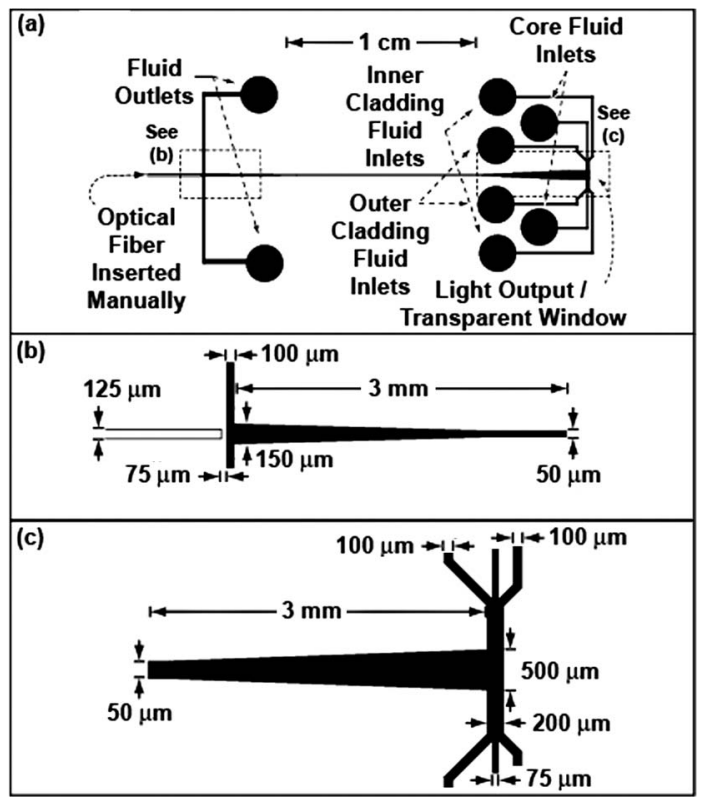

FIG. 1. (a) Diagram of the microfluidic channel used for these experiments. (b) and (c) Enlarged diagrams of the regions of the channel in (a) highlighted by dashed boxes.

Ethylene glycol (neat) and an ethylene glycol:water mixture $(85: 15$ by weight) were used as the fluids for the core $\left(n_{D}=1.431\right)$ and the cladding $\left(n_{D}=1.414\right)$. We chose these solutions for four reasons. First, they do not swell PDMS, ${ }^{11}$ and therefore do not affect the dimensions of the microchannels. Second, the refractive indices of the core and cladding fluids are greater than that of PDMS $\left(n_{D}=1.405\right)$. Third, the fluids are miscible and, therefore, will mix diffusively. Fourth, many organic dyes are soluble in ethylene glycol.

We demonstrated the ability of this system to split a single input beam $(\lambda=780 \mathrm{~nm})$ into two output beams with equal intensities [Fig. 2(a)]. We believe that a contributor to this performance is the smoothness of the flowing fluid stream and smoothness of transition from a single waveguide to two equivalent waveguides. (This smooth gradient at the junction contrasts with sharp boundaries in most solid-core, solid-cladding optical splitters. ${ }^{12}$ ) The angle of the split of the waveguides was estimated to be $<0.5^{\circ}$. We estimated the half-angle of the split by calculating $\arctan (x / y)$, where $x$ was the distance along the length of the channel where the refractive index contrast between the center of one core and the center of the channel $\left(\Delta n=n_{\max }-n_{\min }\right)$ was $\sim 0.001$ and $y$ was the distance along the width of the channel from $n_{\max }$ to $n_{\text {min }}$. This angle is tunable by adjusting the flow rates or geometry of the microfluidic channel.

We modeled the effect of diffusive mixing on the profile of the refractive index along the length of the central, narrow $(50 \mu \mathrm{m})$ region of the microfluidic network to determine how the rates of flow of the liquid components affect this profile [Fig. 2(b)]. The refractive index of the core and cladding streams is directly proportional to the concentration of the components of the liquids. ${ }^{13}$ A gradient in the contrast of the refractive index $\left(\Delta n=n_{\text {core }}-n_{\text {cladding }}\right)$ develops as the fluids flow through the channel due to diffusion of water from the cladding fluid into the core fluid and ethylene glycol from the core fluid into the cladding fluid. To a first approximation, this gradient can be estimated by considering only this mutual diffusion, independent of the composition of the

Downloaded 23 May 2006 to 18.63 .3 .58 . Redistribution subject (a)

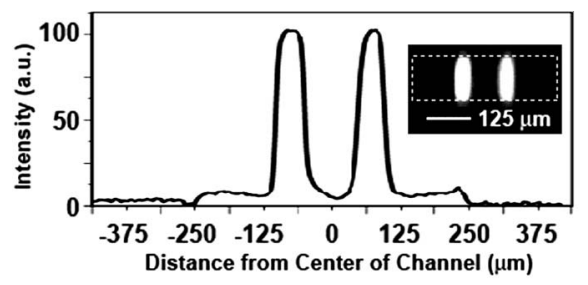

(b)

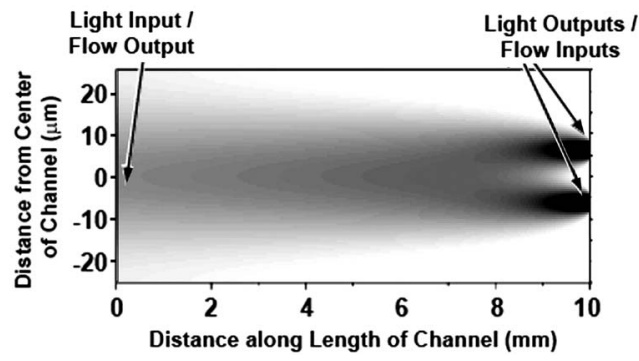

FIG. 2. (a) Plot of the profile of the intensity of light output as a function of distance from the center of the channel. The light $(\lambda=780 \mathrm{~nm})$ was coupled into the $L^{2}$ waveguide shown in Fig. 1 from a single-mode optical fiber. The rate of flow of the core fluids was $2.5 \mu \mathrm{l} / \mathrm{min}$, of the central cladding fluids was $5 \mu \mathrm{l} / \mathrm{min}$, and of the outer cladding fluids was $10 \mu \mathrm{l} / \mathrm{min}$. (Inset) Optical micrograph of light exiting the microfluidic channel, viewed through the transparent window. The dashed box shows the walls of the channel. (b) Contour plot of the refractive index as a function of the distance from the center of the width of the channel and of the distance along the length of the channel. The gradient of gray scale from black to white indicates values of the refractive index from 1.431 to 1.414 . Only the main portion of the waveguide $(1 \mathrm{~cm} \times 0.005 \mathrm{~cm}, l \times w)$ is simulated.

cladding and core fluids; we estimated the diffusion coefficient for this scenario to be $\sim 5 \times 10^{-6} \mathrm{~cm}^{2} / \mathrm{s}$ by taking a value representative of 9:1 (ethylene glycol:water) composition. ${ }^{14}$ This model indicates that diffusion across the width of the central cladding stream separating the two core fluids over the length $(1 \mathrm{~cm})$ of this narrow region of the channel occurs more rapidly than the residence time ( $\tau=$ ratio of the volume of the channel to the rate of flow) of the liquids in this region, for rates of flow $<5 \mu \mathrm{L} / \mathrm{min}$ $[\tau>0.75 \mathrm{~s}$ in channels with dimensions of $0.005 \mathrm{~cm}$ $\times 0.0125 \mathrm{~cm} \times 1 \mathrm{~cm}(w \times h \times l)]$. This diffusion eliminates the optical separation of the core streams in this region.

Slow rates of flow $(<10 \mu \mathrm{L} / \mathrm{min}, \tau>0.375 \mathrm{~s})$ for the core and central cladding stream were necessary to obtain equal splitting of the input beam. High rates of flow ( $>20 \mu \mathrm{L} / \mathrm{min}, \tau<0.19 \mathrm{~s}$ ) resulted in unequal intensities of the output beams from each of the liquid cores. We believe that the most plausible explanation for this observation is that at high rates of flow, the two core fluids and the central cladding fluid do not mix enough to form a single $L^{2}$ waveguide in the region of the light input (and the fluid exit). As a result of the incomplete diffusional mixing, a liquid cladding still separates the two cores at the light input. Imperfections in the alignment of the input solid fiber with respect to these two cores translate into unequal intensities of light coupled into each core. Unequal intensities propagate in individual waveguides and define output intensities observed. Modeling supported this hypothesis of incomplete diffusional mixing.

We exploit the fact that optical properties of the individual core fluids can be separately tuned using streams of liquids containing different dyes. These dye molecules absorb light of specific wavelengths, and their inclusion, therefore, was the basis for a simultaneous, two-color, subtractive wavelength filter. We prepared $L^{2}$ waveguides composed of AIP license or copyright, see http://apl.aip.org/apl/copyright.jsp 

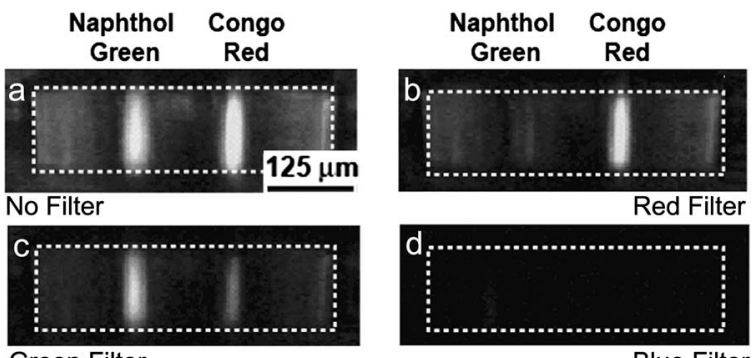

Green Filter

Blue Filter

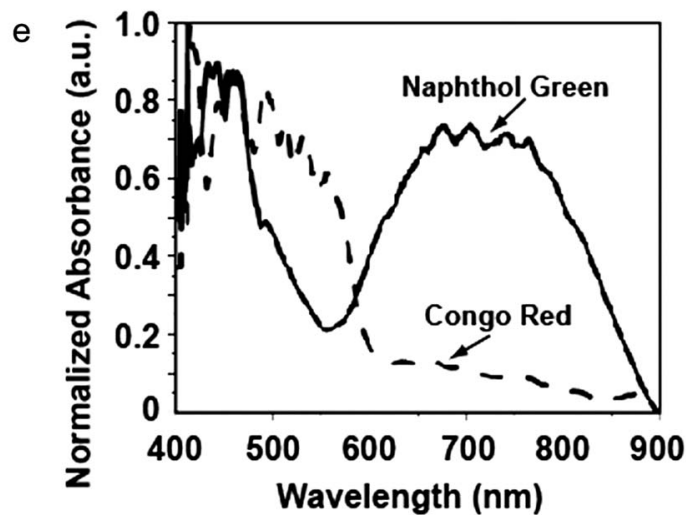

FIG. 3. (a) Optical micrographs of the cross section of the channel viewed through the transparent window. The dashed box shows the walls of the microfluidic channel. The rate of flow of the core fluids was $5 \mu \mathrm{l} / \mathrm{min}$, of the inner cladding fluids was $10 \mu \mathrm{l} / \mathrm{min}$, and of the outer cladding fluids was $20 \mu \mathrm{l} / \mathrm{min}$. The image in (a) was taken without a color filter. The images in (b-d) were taken with the color filter as listed above the image. A small amount of red light was observed in (c) when viewed through the green filter because congo red absorbs light of $\lambda<600 \mathrm{~nm}$ and the green filter transmits wavelengths of $\lambda=600-650 \mathrm{~nm}$. (e) Plot of normalized absorbance (arbitrary units) as a function of wavelength of the light exiting each waveguide.

liquid cores containing dissolved congo red and naphthol green $(1 \mathrm{mM})$. A tungsten lamp, coupled to the $L^{2}$ waveguides with a multimode optical fiber, provided a source of white light. Figure 3(a) is an image of the output beams from each of the dye-doped, $L^{2}$ waveguides. We used color filters (Edmund Industrial Optics, NJ) to show that the light exiting the device had the expected wavelength distribution [Figs. 3(b)-3(d)]. We also measured the absorbance spectrum using a fiber-coupled UV-Vis spectrometer (Spectral Instruments, Inc., Tucson, AZ) to confirm the transmitted spectral range for each dye [Fig. 3(e)].

We did not observe significant diffusive mixing of the dyes along the length of the channel because of the relatively low diffusion constants of the large dye molecules (e.g., compare $2 \times 10^{-6} \mathrm{~cm}^{2} / \mathrm{s}$ for congo $\mathrm{red}^{15}$ vs 11.7 $\times 10^{-6} \mathrm{~cm}^{2} / \mathrm{s}$ for ethylene glycol ${ }^{14}$ in water). As a result, the light contained within one core fluid could be filtered largely independently from the light contained in the second core. The liquid core of the region of the device forming a single waveguide at the light input of the filter contains both red and green dyes, thus, resulting in some absorptive loss in both parts of the spectrum simultaneously.

Optical devices fabricated from $L^{2}$ waveguides have three differences from those fabricated from solid-state optical waveguides. (1) It is easy to fabricate low-loss optical waveguides that split smoothly $\left(\theta_{\text {split }}<0.5^{\circ}\right)$, eliminating sharp discontinuities in the index of refraction typical of solid-state splitters. Solid-state devices require the use of high resolution lithographic tools to generate waveguides

Downloaded 23 May 2006 to 18.63.3.58. Redistribution subject that split with a small angle ${ }^{9}$ and require substantial effort and expense to generate angles $<2^{\circ}$ between the two cores that have optically smooth edges (edge roughness $<500 \mathrm{~nm}$ ). (2) It is possible to control the residence time of the liquids in the channel and the separation between the two liquid cores in real time. This capability determines the extent of diffusive mixing of the $L^{2}$ waveguide components, and thus the refractive index gradient. (3) The dye molecules dissolved in the liquid core can be used to filter wavelengths of light from a white light source. Since the dyes are continually replaced in the flowing waveguide, photobleaching can be minimized. The frequency distribution of the filtered light can also be easily selected by changing dyes.

This system has four significant disadvantages: (1) the syringe pumps used to drive the flow of the liquids make the footprint of the complete system large $\left(>100 \mathrm{~cm}^{2}\right)$; (2) the devices require a constant supply of both the core and the cladding fluids (e.g., at $20 \mu \mathrm{l} / \mathrm{min}, \sim 30 \mathrm{ml}$ of fluid would be necessary to run one stream of the device for $24 \mathrm{~h}$ ); (3) the fluid components of the $L^{2}$ waveguides as described here cannot be easily recycled because they are miscible; (4) the liquid-liquid interfaces in the devices are susceptible to mechanical and thermal perturbations.

This type of $1 \times 2$ optical splitter can, in principle, be expanded laterally to include additional $L^{2}$ waveguides within the same system to create a $l \times n$ splitter, where $n$ $>2$. This capability would enable the simultaneous splitting and filtering of a single, white-light source into many independent, multicolor light sources. The absorbance properties of the liquid cores can be tuned dynamically across the spectrum of visible wavelengths because a large number of organic dyes are soluble in ethylene glycol. We believe the tunable optical properties of these devices could be useful for on-chip analysis where optical excitation and detection of light of specific wavelengths is necessary.

This work was supported by DARPA as a subaward from the California Institute of Technology. The authors thank Dr. J. Christopher Love for helpful discussions.

${ }^{1}$ D. B. Wolfe, R. S. Conroy, P. Garstecki, M. A. Fischbach, K. E. Paul, M. Prentiss, and G. M. Whitesides, Proc. Natl. Acad. Sci. U.S.A. 101, 12434 (2004).

${ }^{2}$ D. B. Wolfe, R. S. Conroy, J. C. Love, B. D. Gates, M. G. Prentiss, and G. M. Whitesides, Appl. Phys. Lett. 84, 1623 (2004).

${ }^{3}$ K. B. Mogensen, Y. C. Kwok, J. C. T. Eijkel, N. J. Petersen, A. Manz, and J. P. Kutter, Anal. Chem. 75, 4931 (2003).

${ }^{4}$ Y. B. Ovchinnikov, Opt. Commun. 220, 229 (2003).

${ }^{5}$ M. Blahut, Proc. SPIE 5028, 90 (2003).

${ }^{6}$ C.-H. Chang and W.-S. Wang, Opt. Quantum Electron. 28, 1371 (1996). ${ }^{7}$ O. J. A. Schueller, X.-M. Zhao, G. M. Whitesides, S. P. Smith, and M. Prentiss, Adv. Mater. 11, 37 (1999).

${ }^{8}$ J. C. McDonald, D. C. Duffy, J. R. Anderson, D. T. Chiu, H. Wu, O. J. Schueller, and G. M. Whitesides, Electrophoresis 21, 27 (2000).

${ }^{9} \mathrm{~T}$. Tamir, Guided-Wave Optoelectronics, 2nd ed. (Springer, New York, 1998).

${ }^{10}$ See EPAPS Document No. E-APPLAB-87-044543 for a photograph with the details of the experimental layout. This document can be reached through a direct link in the article's HTML reference section or via the EPAPS homepage (http://www.aip.org/pubservs/epaps.html).

${ }^{11}$ J. N. Lee, C. Park, and G. M. Whitesides, Anal. Chem. 75, 6544 (2003).

${ }^{12}$ R. G. Hunsperger, Integrated Optics. Theory and Technology (Springer, New York, 2002).

${ }^{13}$ D. R. Lide, CRC Handbook of Chemistry and Physics, 84th ed. (CRC Press, Boca Raton, FL, 2003).

${ }^{14}$ G. Ternstrom, A. Sjostrand, G. Aly, and A. Jernqvist, J. Chem. Eng. Data 41, 876 (1996).

${ }^{15}$ C. Hahn, S. Kaiser, and A. Wokaun, Tenside Surfactants Deterg. 33, 209 (1996). 